\title{
Quadratic and Copositive Lyapunov Functions and the Stability of Positive Switched Linear Systems
}

\author{
Oliver Mason and Robert Shorten
}

\begin{abstract}
We present some new results concerning the stability of positive switched linear systems. In particular, we present a necessary and suf£cient condition for the existence of copositive linear Lyapunov functions for switched systems with two constituent linear time-invariant (LTI) systems. We also extend some recent results on quadratic stability for positive switched linear systems.
\end{abstract}

\section{INTRODUCTION}

Understanding the stability properties of dynamic systems whose states are confned to the positive orthant is of importance for numerous practical applications. Systems of this type are generally referred to as positive systems and arise frequently in areas such as Biology, Communications, Probability and Economics. In particular, many applications in Communication networks involve algorithms that lead to extremely complex positive systems, typically involving signifcant nonlinearity, abrupt parameter switching, and state resets. These applications, which include networks employing TCP and other congestion control applications [16], synchronisation problems [6], wireless power control applications [11], and applications of learning automata to distributed coloring problems [7], typically require advanced analysis tools to prove their stability and convergence properties. Notwithstanding the widespread applications of positive systems, the stability of switched and nonlinear positive system has only attracted major interest from the systems theory community in the relatively recent past [4]. In this paper, we continue this line of work, focussing on questions in the stability of positive switched linear systems. Specifcally, we consider the existence of copositive linear Lyapunov functions, de£ned below, and summarise the work recently reported in [9], providing an elegant necessary and suffcient condition for determining when such a function exists for a class of positive switched systems. For full proofs of these results, the reader should consult [9]. We shall also consider the existence of common quadratic Lyapunov functions (CQLFs) for positive switched systems and extend some recent results on quadratic stability for this class of systems. At the end of the paper, we highlight some possible directions for future work in this area.

\section{NotATion AND MATHEMATICAL BACKGROUND}

Throughout, $\mathbb{R}$ denotes the £eld of real numbers, $\mathbb{R}^{n}$ stands for the vector space of all $n$-tuples of real numbers and $\mathbb{R}^{m \times n}$ is the space of $m \times n$ matrices with real entries. For $x$ in $\mathbb{R}^{n}, x_{i}$ denotes the $i^{t h}$ component of $x$, and the notation $x \succ 0(x \succeq 0)$ means that $x_{i}>0\left(x_{i} \geq 0\right)$ for $1 \leq i \leq n$. The notations $x \prec 0$ and $x \preceq 0$ are de£ned in the obvious manner. $\mathbb{R}_{+}^{n}$ denotes the positive orthant of $\mathbb{R}^{n}, \mathbb{R}_{+}^{n}=\left\{x \in \mathbb{R}^{n}: x \succ 0\right\}$. Similarly, for a matrix $A$ in $\mathbb{R}^{n \times n}, a_{i j}$ denotes the element in the $(i, j)$ position of $A$, and $A \succ 0(A \succeq 0)$ means that $a_{i j}>0\left(a_{i j} \geq 0\right)$ for $1 \leq i, j \leq n$.

We write $A^{T}$ for the transpose of $A$ and we shall slightly abuse notation by writing $A^{-T}$ for the inverse of $A^{T}$. For $P$ in $\mathbb{R}^{n \times n}$ the notation $P>0$ means that the matrix $P$ is positive defnite. A matrix $A \in \mathbb{R}^{n \times n}$ is said to be Hurwitz if all of the eigenvalues of $A$ lie in the open left half of the complex plane.

For a real number $x$ we defne the function $\operatorname{sign}(x)$ by

$$
\operatorname{sign}(x)= \begin{cases}1 & \text { if } x>0 \\ 0 & \text { if } x=0 \\ -1 & \text { if } x<0\end{cases}
$$

Note that if a matrix $A \in \mathbb{R}^{n \times n}$ is Hurwitz, then $\operatorname{sign}(\operatorname{det}(A))=(-1)^{n}$.

Throughout this paper, we shall be concerned with the uniform asymptotic stability, under arbitrary switching, of switched positive linear systems $\dot{x}=$ $A(t) x, A(t) \in\left\{A_{1}, \ldots, A_{m}\right\}$ where each constituent LTI system, $\Sigma_{A_{i}}: \dot{x}=A_{i} x$ is a positive system [2]. Whenever we speak of the asymptotic stability of a switched linear system, uniform asymptotic stability under arbitrary switching is to be understood. Before proceeding, we shall now recall some basic facts about positive LTI systems and their stability.

Positive LTI Systems and Metzler Matrices

The LTI system

$$
\Sigma_{A}: \dot{x}(t)=A x(t), \quad x(0)=x_{0}
$$


is said to be positive if $x_{0} \succeq 0$ implies that $x(t) \succeq 0$ for all $t \geq 0$. Basically, if the system starts in the non-negative orthant of $\mathbb{R}^{n}$, it remains there for all time. See [2] for a description of the basic theory and several applications of positive linear systems.

It is well-known [2] that the system $\Sigma_{A}$ is positive if and only if the off-diagonal entries of the matrix $A$ are non-negative. Matrices of this form are known as Metzler matrices, and can be written $A=N-\alpha I$ for $N \succeq 0$ and $\alpha \geq 0$.

There are a number of equivalent conditions for a Metzler matrix to be Hurwitz [5], [1]. The following result records two of these conditions which are relevant for the work of this paper.

Theorem 2.1: Let $A \in \mathbb{R}^{n \times n}$ be Metzler. Then the following are equivalent:

(i) $A$ is Hurwitz;

(ii) There is some vector $v \succ 0$ in $\mathbb{R}^{n}$ with $A v \prec 0$;

(iii) $A^{-1} \preceq 0$.

\section{Convex Cones and Separation Theorems}

Much of the work presented later in the paper is concerned with determining conditions for the intersection of two convex cones in $\mathbb{R}^{n}$. Recall that a set $\Omega$ in $\mathbb{R}^{n}$ is a convex cone if for all $x, y \in \Omega$, and all $\lambda \geq 0, \mu \geq 0$ in $\mathbb{R}, \lambda x+\mu y$ is in $\Omega$. The convex cone $\Omega$ is said to be open (closed) if it is open (closed) with respect to the usual Euclidean topology on $\mathbb{R}^{n}$. For an open convex cone $\Omega$, we denote the closure of $\Omega$ by $\bar{\Omega}$.

Given a set of points, $\left\{x_{1}, \ldots, x_{m}\right\}$ in $\mathbb{R}^{n}$, we shall use the notation $C O\left(x_{1}, \ldots, x_{m}\right)$ to denote the convex hull of $x_{1}, \ldots, x_{m}$. Formally $C O\left(x_{1}, \ldots, x_{m}\right)$ is the set:

$$
\left\{\sum_{i=1}^{m} \alpha_{i} x_{i}: \alpha_{i} \geq 0,1 \leq i \leq m \text {, and } \sum_{i=1}^{m} \alpha_{i}=1\right\} .
$$

The theory of Enite-dimensional convex sets is a well established branch of mathematical analysis [12]. In the next section, we shall make use of the following special case of more general results [12] on the existence of separating hyperplanes for disjoint convex cones.

Theorem 2.2: Let $\Omega_{1}, \Omega_{2}$ be open convex cones in $\mathbb{R}^{n}$. Suppose that $\overline{\Omega_{1}} \cap \overline{\Omega_{2}}=\{0\}$. Then there is some vector $v \in \mathbb{R}^{n}$ such that

$$
v^{T} x<0 \text { for all } x \in \Omega_{1}
$$

and

$$
v^{T} x>0 \text { for all } x \in \Omega_{2} .
$$

\section{Common Linear Copositive Lyapunov FUNCTIONS}

In this section, we describe a necessary and suffcient condition for a pair of asymptotically stable positive LTI systems to have a common linear copositive Lyapunov function, and discuss a number of implications of this result. First of all, we present some preliminary defnitions and results concerning linear copositive Lyapunov functions.

Preliminaries on Linear Copositive Lyapunov Functions

The linear function $V(x)=v^{T} x$ defnes a linear copositive Lyapunov function for the positive LTI system $\Sigma_{A}$ if and only if the vector $v \in \mathbb{R}^{n}$ satis£es:

(i) $v \succ 0$;

(ii) $A^{T} v \prec 0$.

It follows from Theorem 2.1 that a positive LTI system is asymptotically stable if and only if it has a linear copositive Lyapunov function. The primary contribution of this paper is to derive a simple algebraic necessary and suffcient condition for a pair of asymptotically stable positive LTI systems, $\Sigma_{A_{1}}$, $\Sigma_{A_{2}}$ to have a common linear copositive Lyapunov function $V(x)=v^{T} x$, where $v \succ 0$ and $A_{i}^{T} v \prec 0$ for $i=1,2$. This condition is given in Theorem 3.2 below and our derivation will be based on the following preliminary result, whose proof can be found in [9].

Theorem 3.1: Let $A_{1}, A_{2} \in \mathbb{R}^{n \times n}$ be Metzler, Hurwitz matrices such that there exists no non-zero vector $v \succeq 0$ with $A_{i}^{T} v \preceq 0$ for $i=1,2$. Then there exist $w_{1} \succ 0, w_{2} \succ 0$ in $\mathbb{R}^{n}$ such that

$$
A_{1} w_{1}+A_{2} w_{2}=0
$$

\section{Common Linear Copositive Lyapunov Functions}

Before stating the main result of this section, we need to introduce some notation. Given $A \in \mathbb{R}^{n \times n}$ and an integer $i$ with $1 \leq i \leq n, A^{(i)}$ denotes the $i^{t h}$ column of $A$. Thus, $A^{(i)}$ denotes the vector in $\mathbb{R}^{n}$ whose $j^{t h}$ entry is $a_{j i}$ for $1 \leq j \leq n$.

For a positive integer $n$, we denote the set of all mappings $\sigma:\{1, \ldots, n\} \rightarrow\{1,2\}$ by $\mathcal{C}_{n, 2}$. Now, given two matrices $A_{1}, A_{2}$ in $\mathbb{R}^{n \times n}$ and a mapping $\sigma \in \mathcal{C}_{n, 2}, A_{\sigma}\left(A_{1}, A_{2}\right)$ denotes the matrix

$$
\left(A_{\sigma(1)}^{(1)} A_{\sigma(2)}^{(2)} \ldots A_{\sigma(n)}^{(n)}\right) .
$$

Thus, $A_{\sigma}\left(A_{1}, A_{2}\right)$, is the matrix in $\mathbb{R}^{n \times n}$ whose $i^{t h}$ column is the $i^{t h}$ column of $A_{\sigma(i)}$ for $1 \leq i \leq n$. We shall denote the set of all matrices that can be formed in this way by $\mathcal{S}\left(A_{1}, A_{2}\right)$.

$$
\mathcal{S}\left(A_{1}, A_{2}\right)=\left\{A_{\sigma}\left(A_{1}, A_{2}\right): \sigma \in \mathcal{C}_{n, 2}\right\} .
$$


Theorem 3.2: Let $A_{1}, A_{2}$ be Metzler, Hurwitz matrices in $\mathbb{R}^{n \times n}$. Then the following statements are equivalent:

(i) The positive LTI systems $\Sigma_{A_{1}}, \Sigma_{A_{2}}$ have a common linear copositive Lyapunov function;

(ii) The Enite set $\mathcal{S}\left(A_{1}, A_{2}\right)$ consists entirely of Hurwitz matrices.

\section{Proof:}

(i) $\Rightarrow$ (ii): As $\Sigma_{A_{1}}, \Sigma_{A_{2}}$ have a common linear copositive Lyapunov function, there is some vector $v \succ 0$ in $\mathbb{R}^{n}$ with $v^{T} A_{i} \prec 0$ for $i=1,2$. This immediately implies that $v^{T} A_{i}^{(j)}<0$ for $i=1,2$, $1 \leq j \leq n$ and hence we have that

$$
v^{T} A \prec 0 \text { for all } A \in \mathcal{S}\left(A_{1}, A_{2}\right) .
$$

Now note that as $A_{1}, A_{2}$ are Metzler, all matrices belonging to the set $\mathcal{S}\left(A_{1}, A_{2}\right)$ are also Metzler. It follows immediately from (3) and the standard properties of Metzler matrices that each matrix in $\mathcal{S}\left(A_{1}, A_{2}\right)$ must be Hurwitz.

(ii) $\Rightarrow\left(\right.$ i): We shall show that if $\Sigma_{A_{1}}, \Sigma_{A_{2}}$ do not have a common linear copositive Lyapunov function, then at least one matrix belonging to the set $\mathcal{S}\left(A_{1}, A_{2}\right)$ must be non-Hurwitz.

First of all, suppose that there is no non-zero vector $v \succeq 0$ with $v^{T} A_{i} \preceq 0$ for $i=1,2$. It follows from Theorem 3.1 that there are vectors $w_{1}, w_{2}$ such that $w_{1} \succ 0, w_{2} \succ 0$ and

$$
A_{1} w_{1}+A_{2} w_{2}=0 .
$$

As $w_{1} \succ 0, w_{2} \succ 0$, there is some positive defnite diagonal matrix $D=\operatorname{diag}\left(d_{1}, d_{2}, \ldots, d_{n}\right)$ in $\mathbb{R}^{n \times n}$ with $w_{2}=D w_{1}$. It follows from (4) that, for this $D$,

$$
\operatorname{det}\left(A_{1}+A_{2} D\right)=0 \text {. }
$$

Now, for an $n$-tuple, $\left(d_{1}, \ldots, d_{n}\right)^{T} \in \mathbb{R}^{n}$ and a mapping $\sigma \in \mathcal{C}_{n, 2}$, we shall use $\left(d_{1}, \ldots, d_{n}\right)^{\sigma-1}$ to denote the product of $d_{1}, \ldots, d_{n}$ given by

$$
\left(d_{1}, \ldots, d_{n}\right)^{\sigma-1}=\prod_{i=1}^{n} d_{i}^{\sigma(i)-1} .
$$

In terms of this notation, the polynomial $\operatorname{det}\left(A_{1}+\right.$ $\left.A_{2} D\right)$ in the variables $d_{1}, \ldots, d_{n}$ is given by

$$
\sum_{\sigma \in \mathcal{C}_{n, 2}} \operatorname{det}\left(A_{\sigma}\left(A_{1}, A_{2}\right)\right)\left(d_{1}, \ldots, d_{n}\right)^{\sigma-1}
$$

Now if all matrices in the set $\mathcal{S}\left(A_{1}, A_{2}\right)$ were Hurwitz, then $\operatorname{det}\left(A_{\sigma}\left(A_{1}, A_{2}\right)\right)>0$ for all $\sigma \in \mathcal{C}_{n, 2}$ if $n$ is even and $\operatorname{det}\left(A_{\sigma}\left(A_{1}, A_{2}\right)\right)<0$ for all $\sigma \in \mathcal{C}_{n, 2}$ if $n$ is odd. In either case, this would contradict (5) which implies that there are positive real numbers $d_{1}, \ldots, d_{n}$ for which

$$
\sum_{\sigma \in \mathcal{C}_{n, 2}} \operatorname{det}\left(A_{\sigma}\left(A_{1}, A_{2}\right)\right)\left(d_{1}, \ldots, d_{n}\right)^{\sigma-1}=0 .
$$

Hence, there must exist at least one $\sigma \in \mathcal{C}_{n, 2}$ for which $A_{\sigma}\left(A_{1}, A_{2}\right)$ is non-Hurwitz.

For the remainder of the proof, we shall assume that the dimension $n$ is even. In this case, for a Hurwitz $A \in \mathbb{R}^{n \times n}, \operatorname{det}(A)>0$. The case of odd $n$ follows in an identical manner.

We have shown that if $\overline{\mathcal{V}_{A_{1}}} \cap \overline{\mathcal{V}_{A_{2}}}=\{0\}$, then at least one matrix belonging to $\mathcal{S}\left(A_{1}, A_{2}\right)$ must be nonHurwitz. In fact, we have shown that $\operatorname{det}(A)<0$ for at least one $A$ belonging to $\mathcal{S}\left(A_{1}, A_{2}\right)$. Next suppose that there is some non-zero $v \succeq 0$ in $\overline{\mathcal{V}_{A_{1}}} \cap \overline{\mathcal{V}_{A_{2}}}$ but that the intersection of the open cones

$$
\mathcal{V}_{A_{1}} \cap \mathcal{V}_{A_{2}}
$$

is empty.

Now, denote by $\mathbf{1}_{n}$ the matrix in $\mathbb{R}^{n \times n}$ consisting entirely of ones $\left(\mathbf{1}_{n}(i, j)=1\right.$ for $\left.1 \leq i, j \leq n\right)$ and for all $\epsilon>0$, write $A_{i}(\epsilon)=A_{i}+\epsilon \mathbf{1}_{n}$ for $i=1,2$. Then it is straightforward to see that

$$
\overline{\mathcal{V}_{A_{1}(\epsilon)}} \cap \overline{\mathcal{V}_{A_{2}(\epsilon)}}=\{0\}
$$

for all $\epsilon>0$. Thus, if we choose any $\epsilon>0$ suffciently small to ensure that $A_{1}(\epsilon)$ and $A_{2}(\epsilon)$ are Hurwitz and Metzler, it follows from the above argument that there must be at least one non-Hurwitz matrix in the set $\mathcal{S}\left(A_{1}(\epsilon), A_{2}(\epsilon)\right)$. A limiting argument now shows that at least one matrix in the set $\mathcal{S}\left(A_{1}, A_{2}\right)$ is non-Hurwitz. This completes the proof of the theorem.

We now present a simple example to illustrate the use of the above theorem.

Example 3.1: Consider the Metzler, Hurwitz matrices in $\mathbb{R}^{2 \times 2}$ given by

$$
\begin{aligned}
& A_{1}=\left(\begin{array}{ll}
-0.7125 & 0.7764 \\
0.5113 & -0.9397
\end{array}\right), \\
& A_{2}=\left(\begin{array}{ll}
-1.3768 & 0.8066 \\
0.9827 & -1.3738
\end{array}\right) .
\end{aligned}
$$

Then it is easy to see that $\mathcal{S}\left(A_{1}, A_{2}\right)$ consists entirely of Hurwitz matrices. It follows from Theorem 3.2 that the systems $\Sigma_{A_{1}}, \Sigma_{A_{2}}$ have a common linear copositive Lyapunov function. In fact, for $v=$ $(1.1499,1.1636)^{T}$, it can be checked that $A_{i}^{T} v \prec 0$ for $i=1,2$.

\section{Remarks:}

(i) Note that the result of Theorem 3.2 relates the existence of a common Lyapunov function for a pair of positive LTI systems, and the uniform asymptotic stability of the associated switched linear system, to the stability of a Enite set of positive LTI systems. Formally, the existence of a common linear copositive Lyapunov function 
for $\Sigma_{A_{1}}, \Sigma_{A_{2}}$ is equivalent to the stability of each of the $2^{n}$ positive LTI systems, $\Sigma_{A}$ for $A \in \mathcal{S}\left(A_{1}, A_{2}\right)$. Of course, it follows that the asymptotic stability of this £nite family of systems is suffcient for the uniform asymptotic stability of the switched system $\dot{x}=A(t) x$, $A(t) \in\left\{A_{1}, A_{2}\right\}$.

(ii) A common linear copositive Lyapunov function for $\Sigma_{A_{1}}, \Sigma_{A_{2}}$ will also defne a linear copositive Lyapunov function for each of the systems $\Sigma_{A}$ with $A \in \mathcal{S}\left(A_{1}, A_{2}\right)$.

(iii) In the proof of Theorem 3.2, the non-existence of a common linear copositive Lyapunov function is related to the existence of a diagonal matrix $D>0$ such that $A_{1}+A_{2} D$ is singular. It is interesting to compare this with the recent result in [10], which established that the non-existence of a common diagonal Lyapunov function for a pair of positive LTI systems implied the existence of a diagonal $D>0$ such that $A_{1}+D A_{2} D$ is singular. The precise relationship between copositive Lyapunov functions, diagonal Lyapunov functions and quadratic Laypunov functions for general switched positive linear systems is in itself an interesting question, and the above result may prove useful in clarifying this relationship.

Using the above remarks and Theorem 3.2, we can derive the following result.

Corollary 3.1: Let $A_{1}, A_{2}$ be Metzler, Hurwitz matrices in $\mathbb{R}^{n \times n}$. Then the following statements are equivalent:

(i) There exists a common linear copositive Lyapunov function for the systems $\Sigma_{A_{1}}, \Sigma_{A_{2}}$;

(ii) There is a common linear copositive Lyapunov function for the set of systems

$$
\left\{\Sigma_{A}: A \in C O\left(\mathcal{S}\left(A_{1}, A_{2}\right)\right)\right\}
$$

(iii) All matrices in the convex hull $C O\left(\mathcal{S}\left(A_{1}, A_{2}\right)\right)$ are Hurwitz;

(iv) All matrices in $\mathcal{S}\left(A_{1}, A_{2}\right)$ are Hurwitz.

Proof: (i) $\Rightarrow$ (ii): Suppose that $V(x)=v^{T} x$ is a common linear copositive Lyapunov function for $\Sigma_{A_{1}}, \Sigma_{A_{2}}$. Then it follows that $v^{T} A_{i} \prec 0$ for $i=1,2$ and hence that $v^{T} A_{i}^{(j)}<0$ for $i=1, \ldots, n$, $j=1, \ldots, n$. Thus, $v^{T} A \prec 0$ for all $A \in \mathcal{S}\left(A_{1}, A_{2}\right)$. It follows immediately that $V(x)=v^{T} x$ will defne a linear copositive Lyapunov function for $\Sigma_{A}$ for all $A \in C O\left(\mathcal{S}\left(A_{1}, A_{2}\right)\right)$.

(ii) $\Rightarrow$ (iii): By assumption, there exists a vector $v \succ$ 0 in $\mathbb{R}^{n}$ with $v^{T} A \prec 0$ for all $A \in C O\left(\mathcal{S}\left(A_{1}, A_{2}\right)\right)$. But every matrix in $C O\left(\mathcal{S}\left(A_{1}, A_{2}\right)\right)$ is Metzler. It follows immediately that each $A \in C O\left(\mathcal{S}\left(A_{1}, A_{2}\right)\right)$ is Hurwitz. (iii) $\Rightarrow$ (iv): This is trivial as $\mathcal{S}\left(A_{1}, A_{2}\right) \subset$ $C O\left(\mathcal{S}\left(A_{1}, A_{2}\right)\right)$.

(iv) $\Rightarrow$ (i): This follows from Theorem 3.2.

The previous corollary shows that the Hurwitzstability of the Enite collection of matrices $\mathcal{S}\left(A_{1}, A_{2}\right)$ is suffcient to ensure the asymptotic stability under arbitrary switching of the system

$$
\dot{x}=A(t) x \quad A(t) \in C O\left(\mathcal{S}\left(A_{1}, A_{2}\right)\right) .
$$

Also, the equivalence of points (iii) and (iv) above means that the Hurwitz-stability of the set $\mathcal{S}\left(A_{1}, A_{2}\right)$ is necessary and suffcient for the Hurwitz-stability of its convex hull.

A close examination of the proof of Theorem 3.2 shows that the following characterisation of linear copositive Lyapunov function existence also holds.

Corollary 3.2: Let $A_{1}, A_{2} \in \mathbb{R}^{n \times n}$ be Metzler, Hurwitz matrices. Then the systems $\Sigma_{A_{1}}, \Sigma_{A_{2}}$ have a common linear copositive Lyapunov function if and only if

$$
\operatorname{sign}(\operatorname{det}(A))=(-1)^{n}
$$

for all $A \in \mathcal{S}\left(A_{1}, A_{2}\right)$.

\section{QUADRATIC STABILITY FOR POSITIVE SYSTEMS DIFFERING BY RANK ONE}

A popular approach to establishing the asymptotic stability of a switched linear system under arbitrary switching regimes is to search for a common quadratic Lyapunov function (CQLF) for its constituent systems [8], [15]. To date, a number of elegant analytic conditions for CQLF existence for classes of switched systems have appeared in the literature. In particular, for a system $\dot{x}=A(t) x$, $A(t) \in\left\{A_{1}, A_{2}\right\}$ with $\operatorname{rank}\left(A_{2}-A_{1}\right)=1$, it has been established that CQLF existence is equivalent to the matrix product $A_{1} A_{2}$ having no negative real eigenvalues. Formally:

Theorem 4.1: [13], [14] Let $A_{1}, A_{2}$ be two Hurwitz matrices in $\mathbb{R}^{n \times n}$ with $\operatorname{rank}\left(A_{2}-A_{1}\right)=1$. A necessary and suffcient condition for the existence of a CQLF for the LTI systems $\Sigma_{A_{1}}, \Sigma_{A_{2}}$ is that the matrix product $A_{1} A_{2}$ does not have any negative real eigenvalues.

In this section, we shall show that this result has interesting consequences when applied to the particular case of positive switched linear systems.

\section{Second Order Systems}

First, we recall two results on $2 \times 2$ matrices, recently published in [3] that are of relevance in the current context. 
Lemma 4.1: [3] Let $A_{1}, A_{2} \in \mathbb{R}^{2 \times 2}$ be Hurwitz and Metzler. Then the product $A_{1} A_{2}$ has no negative real eigenvalue.

Theorem 4.2: [3] Let $A_{1}, \ldots, A_{m}$ be Hurwitz, Metzler matrices in $\mathbb{R}^{2 \times 2}$. Then the positive switched linear system,

$$
\dot{x}=A(t) x \quad A(t) \in\left\{A_{1}, \ldots, A_{m}\right\},
$$

is uniformly asymptotically stable for arbitrary switching if and only if each of the switched linear systems,

$$
\dot{x}=A(t) x \quad A(t) \in\left\{A_{i}, A_{j}\right\},
$$

for $1 \leq i<j \leq m$ is uniformly asymptotically stable under arbitrary switching.

The next result is an immediate consequence of Theorem 4.1 and Lemma 4.1

Theorem 4.3: Let $A_{1}, A_{2}$ be Hurwitz, Metzler matrices in $\mathbb{R}^{2 \times 2}$ such that $\operatorname{rank}\left(A_{2}-A_{1}\right)=1$. Then the LTI systems $\Sigma_{A_{1}}, \Sigma_{A_{2}}$ have a CQLF, and the switched system $\dot{x}=A(t) x, A(t) \in\left\{A_{1}, A_{2}\right\}$ is uniformly asymptotically stable under arbitrary switching.

Theorem 4.3 shows that a positive switched linear system, $\dot{x}=A(t) x, A(t) \in\left\{A_{1}, A_{2}\right\}$, with $A_{1}, A_{2} \in \mathbb{R}^{2 \times 2}$ Hurwitz and $\operatorname{rank}\left(A_{2}-A_{1}\right)=$ 1 , is always asymptotically stable under arbitrary switching. We shall next use Theorem 4.2 to extend this result to the case of a switched positive linear system with an arbitrary £nite number of constituent systems.

Theorem 4.4: Let $A_{1}, \ldots, A_{m}$ be Hurwitz, Metzler matrices in $\mathbb{R}^{2 \times 2}$, such that $\operatorname{rank}\left(A_{i}-A_{j}\right)=1$ for $1 \leq i<j \leq m$. Then the positive switched linear system

$$
\dot{x}=A(t) x \quad A(t) \in\left\{A_{1}, \ldots, A_{m}\right\},
$$

is asymptotically stable under arbitrary switching.

Proof: From Theorem 4.2, the system (12) is asymptotically stable under arbitrary switching, if and only if each of the associated systems $\dot{x}=A(t) x, A(t) \in$ $\left\{A_{i}, A_{j}\right\}, 1 \leq i<j \leq m$ is asymptotically stable under arbitrary switching. But it follows immediately from Theorem 4.3 that each of these systems has a CQLF and hence is asymptotically stable under arbitrary switching. This completes the proof.

\section{Third Order Systems}

Finally for this section, we shall present an extension of the result of Lemma 4.1 to third order positive systems. In the proof of the following theorem, we use the notation $|A|$ to denote the determinant of the matrix $A$.
Theorem 4.5: Let $A_{1}, A_{2} \in \mathbb{R}^{3 \times 3}$ be Metzler and Hurwitz, and let $\gamma>0$ be any positive real number. Then $\operatorname{det}\left(A_{1} A_{2}+\gamma I\right)>\operatorname{det}\left(A_{1} A_{2}\right)$.

Proof: If we write $B=A_{1} A_{2}$, then the following facts can be easily veri£ed.

(i) $\operatorname{det}(B)>0$;

(ii) $b_{i i}>0$ for $1 \leq i \leq 3$;

(iii) $B^{-1}=A_{2}^{-1} A_{1}^{-1} \succeq 0$.

From (i) and (iii), it follows that, if we write $B_{i i}$ for the principal sub-matrix of $B$ obtained by removing its $i^{\text {th }}$ row and column, then $\operatorname{det}\left(B_{i i}\right) \geq 0$ for $1 \leq$ $i \leq 3$.

Now consider

$\operatorname{det}(B+\gamma I)=\left|\begin{array}{ccc}b_{11}+\gamma & b_{12} & b_{13} \\ b_{21} & b_{22}+\gamma & b_{23} \\ b_{31} & b_{32} & b_{33}+\gamma\end{array}\right|$.

As the determinant is a multi-linear function of the columns of a matrix, we can expand (13) using the frst column to see that

$$
\begin{aligned}
\operatorname{det}(B+\gamma I) & =\left|\begin{array}{ccc}
b_{11} & b_{12} & b_{13} \\
b_{21} & b_{22}+\gamma & b_{23} \\
b_{31} & b_{32} & b_{33}+\gamma
\end{array}\right| \\
& +\gamma\left|\begin{array}{cc}
b_{22}+\gamma & b_{23} \\
b_{32} & b_{33}+\gamma
\end{array}\right| .
\end{aligned}
$$

Now, considering the frst term on the right hand side of (14) and repeating the above process using the second column this time, we fnd that

$$
\left|\begin{array}{ccc}
b_{11} & b_{12} & b_{13} \\
b_{21} & b_{22}+\gamma & b_{23} \\
b_{31} & b_{32} & b_{33}+\gamma
\end{array}\right|
$$

is equal to

$$
\left|\begin{array}{ccc}
b_{11} & b_{12} & b_{13} \\
b_{21} & b_{22} & b_{23} \\
b_{31} & b_{32} & b_{33}+\gamma
\end{array}\right|+\gamma\left|\begin{array}{cc}
b_{11} & b_{13} \\
b_{31} & b_{33}+\gamma
\end{array}\right|
$$

Finally, if we expand the frst term on the right hand side of (15) using its third column we can see that

$$
\operatorname{det}(B+\gamma I)=\operatorname{det}(B)+\gamma \Delta(\gamma)
$$

where

$$
\begin{gathered}
\Delta(\gamma)=\left|\begin{array}{cc}
b_{22}+\gamma & b_{23} \\
b_{32} & b_{33}+\gamma
\end{array}\right| \\
+\left|\begin{array}{cc}
b_{11} & b_{13} \\
b_{31} & b_{33}+\gamma
\end{array}\right|+\left|\begin{array}{ll}
b_{11} & b_{12} \\
b_{21} & b_{22}
\end{array}\right| .
\end{gathered}
$$

Considering the second order determinants in (16) in turn, it follows from points (i), (ii) and (iii) made at the beginning of the proof that

$$
\left|\begin{array}{cc}
b_{22}+\gamma & b_{23} \\
b_{32} & b_{33}+\gamma
\end{array}\right|>\operatorname{det}\left(B_{11}\right) \geq 0
$$


and

$$
\left|\begin{array}{cc}
b_{11} & b_{13} \\
b_{32} & b_{33}+\gamma
\end{array}\right|>\operatorname{det}\left(B_{22}\right) \geq 0
$$

It is now immediate from (16) that

$$
\operatorname{det}\left(A_{1} A_{2}+\gamma I\right)>\operatorname{det}\left(A_{1} A_{2}\right)
$$

as claimed.

It follows immediately from Theorem 4.5 that if $A_{1}, A_{2} \in \mathbb{R}^{3 \times 3}$ are Metzler and Hurwitz, then $A_{1} A_{2}$ cannot have any negative real eigenvalues. Hence, we have the following extension of Theorem 4.3.

Theorem 4.6: Let $A_{1}, A_{2}$ be Hurwitz Metzler matrices in $\mathbb{R}^{3 \times 3}$ with $\operatorname{rank}\left(A_{2}-A_{1}\right)=1$. Then the LTI systems $\Sigma_{A_{1}}, \Sigma_{A_{2}}$ have a CQLF, and the associated positive switched linear system $\dot{x}=A(t) x \quad A(t) \in$ $\left\{A_{1}, A_{2}\right\}$, is uniformly asymptotically stable under arbitrary switching.

\section{CONCLUSIONS}

In this paper we have presented a method for determining whether or not a given switched positive continuous time linear system is asymptotically stable. Our approach is based upon determining verifable conditions for the existence of a common copositive linear Lyapunov function for a pair of positive LTI systems. Future work will involve extending this result to arbitrary £nite sets of such LTI systems, and developing synthesis procedures to exploit our result for the design of stable switched positive systems. We have also extended some recent work on the quadratic stability of positive switched systems. In this connection, future work will focus on investigating the possibilities of obtaining analogous results for higher dimensional systems and for arbitrary £nite families of LTI systems.

Acknowledgements: This work was partially supported by Science Foundation Ireland (SFI) grants 03/RP1/I382 and 04/IN1/I478. Science Foundation Ireland is not responsible for any use of data appearing in this publication.

\section{REFERENCES}

[1] A. Berman and R.J. Plemmon. Non-negative matrices in the mathematical sciences. SIAM classics in applied mathematics, 1994.

[2] L. Farina and S. Rinaldi. Positive Linear Systems. Wiley Interscience Series, 2000.

[3] L. Gurvits, R. Shorten, and O. Mason. Preliminary results on the stability of switched positive linear systems. In Mathematical Theory of Networks and Systems, 2004.

[4] L. Gurvits, R. Shorten, and O. Mason. On the stability of switched positive linear systems. submitted to IEEE Transactions on Automatic Control, 2006.

[5] R. Horn and C. Johnson. Topics in Matrix Analysis. Cambridge University Press, 1991.
[6] A. Jadbabaie, J. Lin, and A. S. Morse. Co-ordination of groups of mobile autonomous agents using nearest neighbour rules. IEEE Transactions on Automatic Control, 48(6):988-1001, 2003.

[7] D. Leith and P. Clifford. Convergence of distributed learning algorithms for optimal wireless channel allocation. In Proceedings of IEEE Conference on Decision And Control, 2006.

[8] Daniel Liberzon and A. Stephen Morse. Basic problems in stability and design of switched systems. IEEE Control Systems Magazine, 19(5):59-70, 1999.

[9] O. Mason and R. Shorten. On linear copositive Lyapunov functions and the stability of switched positive linear systems. submitted to IEEE Transactions on Automatic Control, 2006.

[10] O. Mason and R. Shorten. On the simultaneous diagonal stability of a pair of positive linear systems. Linear Algebra and its Applications, 413:13-23, 2006.

[11] A. Paul, M. Akar, U. Mitra, and M. Safonov. A switched system model for stability analysis of distributed power control algorithms for cellular communications. In Proceedings of American Control Conference, 2004.

[12] R. T. Rockafellar. Convex Analysis. Princeton University Press, 1970.

[13] R. N. Shorten, O. Mason, F. O' Cairbre, and P. Curran. A unifying framework for the SISO Circle Criterion and other quadratic stability criteria. International Journal of Control, 77(1):1-8, 2004.

[14] R. N. Shorten and K. S. Narendra. On common quadratic Lyapunov functions for pairs of stable LTI systems whose system matrices are in companion form. IEEE Transactions on Automatic Control, 48(4):618-621, 2003.

[15] R. N. Shorten, K. S. Narendra, and O. Mason. A result on common quadratic Lyapunov functions. IEEE Transactions on Automatic Control, 48(1):110-113, 2003.

[16] R. N. Shorten, F. Wirth, and D. Leith. A positive systems model of TCP-like congestion control: Asymptotic results. IEEE/ACM Transactions on Networking, 14(3):616-629, 2006. 